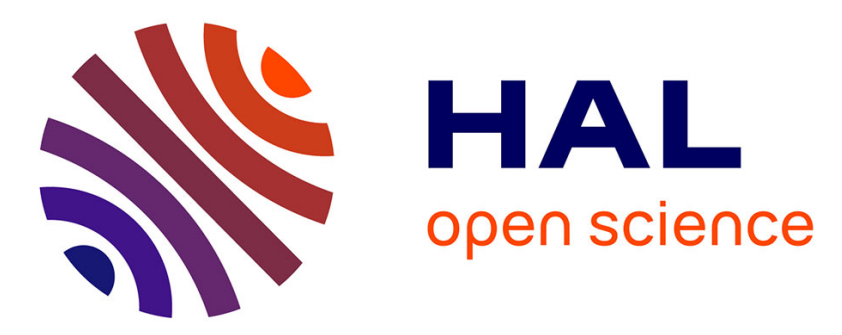

\title{
Digital holography super-resolution for accurate three-dimensional reconstruction of particle holograms
}

Nicolas Verrier, Corinne Fournier

\section{To cite this version:}

Nicolas Verrier, Corinne Fournier. Digital holography super-resolution for accurate three-dimensional reconstruction of particle holograms: Super résolution numérique pour la reconstruction 3D d'hologrammes de particules.. Optics Letters, 2015, 40 (2), pp.217-220. 10.1364/OL.40.000217. ujm-01091509v2

\section{HAL Id: ujm-01091509 https://hal-ujm.archives-ouvertes.fr/ujm-01091509v2}

Submitted on 2 Feb 2015

HAL is a multi-disciplinary open access archive for the deposit and dissemination of scientific research documents, whether they are published or not. The documents may come from teaching and research institutions in France or abroad, or from public or private research centers.
L'archive ouverte pluridisciplinaire $\mathbf{H A L}$, est destinée au dépôt et à la diffusion de documents scientifiques de niveau recherche, publiés ou non, émanant des établissements d'enseignement et de recherche français ou étrangers, des laboratoires publics ou privés. 


\title{
Digital holography super-resolution for accurate three dimensional reconstruction of particle holograms
}

\author{
Nicolas Verrier ${ }^{1}$ and Corinne Fournier ${ }^{1, *}$ \\ ${ }^{1}$ Université de Lyon, F-42023 Saint-Etienne, France, \\ CNRS UMR 5516 Laboratoire Hubert Curien, F-42000 Saint-Etienne, France, \\ Université de Saint-Etienne Jean Monnet, F-42000 Saint-Etienne, France.
}

compiled: November 11, 2014

\begin{abstract}
In-line digital holography is used in many fields to locate and size micro- or nano-objects spread in a volume. To reconstruct simple shaped objects, the optimal approach is to fit an imaging model to accurately estimate their position and their characteristic parameters. Increasing the accuracy of the reconstruction is a big issue in digital holography, particularly when the pixel is large or the signal-to-noise ratio is low. We suggest exploiting the information redundancy of videos to improve the reconstruction of the holograms by jointly estimating the position of the objects and the characteristic parameters. Using synthetic and experimental data we checked experimentally that this approach can improve the accuracy of the reconstruction by a factor more than the square root of the image number.
\end{abstract}

OCIS codes: $\quad$ (090.1995) Holography: Digital holography, (100.3190) Image processing: Inverse problems, (100.6640) Image processing: Superresolution; (100.3010) Image processing: Image reconstruction techniques, (120.3940) Instrumentation, measurement, and metrology: Metrology

The quantitative three-dimensional reconstruction and tracking of micro- or nano-objects spread in a volume is of great interest in many fields of science (e.g. biomedicine, chemical engineering, fluid mechanics). Digital Holography (DH) is a quantitative 3D imaging system that is time-resolved. It is consequently an appropriate tool to study fast 3D phenomena. However, increasing the accuracy of the reconstruction is a major challenge in DH, particularly when the signal to noise ratio is low, the pixel is large (e.g. for high speed cameras) or the numerical aperture is low. In the last case, depth accuracy and lateral accuracy can differ by an order of magnitude [1].

Many numerical methods for DH accuracy improvement have been described in the literature in recent decades. Recently, "pixel Super-Resolution (SR)" approaches, which are generally used for conventional imaging, were used to improve the resolution of reconstructed digital holograms $[2,3]$. These approaches consist of four main steps: recording multiple holographic images that are shifted with respect to one another, image registration, computation of the super-resolved hologram, and holographic reconstruction. This enables a significant gain in resolution. However, as the image is processed as one block $[2,4]$, this is very sensitive to the presence of any objects in the field whose motion differs from that of the objects of interest [5].

\footnotetext{
* corinne.fournier@univ-st-etienne.fr
}

"Inverse Problems" (IP) approaches have also been proposed to more accurately reconstruct digital holograms. Rather than reconstructing the hologram (e.g., using numerical diffraction), they aim to find the simulation (i.e., direct problem) that is closest to the data. This approach is sometimes referred to as Bayesian approach [6] or as compressive holography $[7,8]$. For arbitrarily shaped objects, this approach makes it possible to reconstruct their opacity distribution sampled on a 3-D grid by inverting the hologram formation model using an appropriate regularization $[6,9]$. For parametric objects (described by few parameters) whose diffraction patterns are given by an analytic formula, inverse reconstruction can be performed by maximum likelihood fitting of an imaging model to the data $[10,11]$. This approach is sometimes called "super-resolution" (see e.g., [12]). These IP methods have been shown to be optimal in certain cases $[12,13]$ and lead to almost unsupervised algorithms. To further improve the $3 \mathrm{D}$ reconstruction accuracy of parametric objects, we suggest combining the potential of such IP approach and pixel SR.

The IP approach applied to one image in the stack enables an optimal estimation, and in some cases, reaches Cramer Rao lower bounds $[12,13]$. In the case of a $n_{\text {img }}$ image stack, applying this IP approach to each image and averaging the estimated parameters decreases the standard deviation of the estimator by $\sqrt{n_{\text {img }}}[14]$. However, it has been shown that joint estimation of the parameters leads to more accurate results, particularly since information intercorrelation between parameters is 
non negligible [5]. In that case, the gain in accuracy can be greater than $\sqrt{n_{\text {img }}}$. In the following, without loss of generality, we consider opaque spherical objects. The imaging model is the Thompson model [15] and has four parameters: radius $r$ and 3D position $(x, y, z)$. We now describe the algorithm used to jointly estimate, in a stack of images, the object parameters, which are the transverse shifts of the object $\left\{\Delta x_{i}, \Delta y_{i}\right\}_{i=1: n_{\text {img }}}$, the axial position $z$, and the radius $r$. We then present the results we obtained using this approach on synthetic and experimental holograms and compare them to independent parameter estimation and classical pixel SR reconstruction.

\section{Rough estimation: Matching Pursuit}

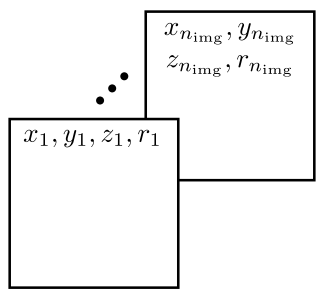

Refinement: Joint Optimization

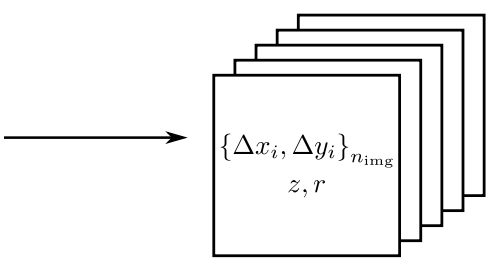

Fig. 1. Joint parameter estimation.

The proposed algorithm (Fig. 1) is a modified version of the unsupervised algorithm proposed by Soulez et al. [10] adapted to the processing of shifted hologram stacks. It performs joint estimation of the shifts $\left\{\Delta x_{i}, \Delta y_{i}\right\}_{i=1: n_{\text {img }}}$ and object parameters $\{z, r\}$. It makes it possible to reconstruct objects outside of the classical field of view and account for irrelevant data regions in the hologram thanks to a weighted mask [16]. It consists of two main steps:

- A rough estimation step, which detects the best matching element in a dictionary of diffraction patterns (computed using a discrete sampling of the parameter space, $\left.\left\{x_{i}, y_{i}, z_{i}, r_{i}\right\}\right)$ for each hologram in the stack. - This step allows estimation of integer pixel shifts between holograms and coarse estimation of $z$ and $r$. The latter are computed by averaging all the estimates of the stack.

- A local joint optimization step, which improves the estimate by fitting the parametric model. This step leads to accurate measurement of sub-pixel shifts, $z$ and $r$.

This algorithm can be used iteratively when several objects of interest are in the field of view. In that case, a cleaning step, which subtracts the detected pattern from the hologram stack, allows the signal-to-noise ratio of the remaining patterns to be increased.

To demonstrate the advantages of the proposed SR scheme, the method was validated in both numerical and bench-top experiments. Fig. 2 shows the experimental

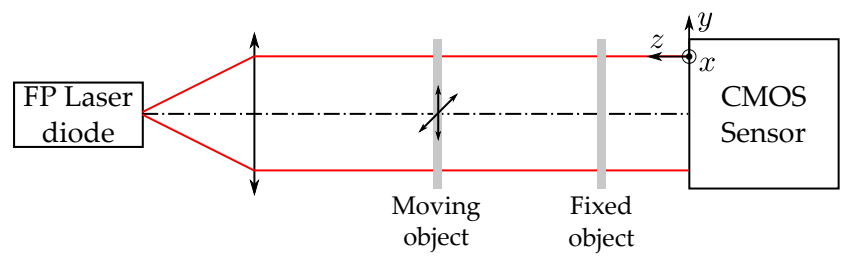

Fig. 2. (Color online) Experimental configuration for superresolution of particle holograms.

configuration, which consists in a classical in-line digital holography arrangement. The light emitted by a $\lambda=662 \mathrm{~nm}, 75 \mathrm{~mW}$ fiber pigtailed (FP) laser diode (Coherent OBIS 660 LX $\AA$ ) was collimated to act as the reference beam. The sample is represented by two objects. The first is moving, while the other remains stationary. Interference between the reference and the object beams are recorded by a high-speed 1280x1024 pixel CMOS sensor (NAC MEMRECAM K4@), with a pixel pitch of $21.7 \mu \mathrm{m}$ and a fill-factor (i.e., active area over total area of pixel) of 0.7 at a frame-rate of $1000 \mathrm{~Hz}$. SR is performed by jointly optimizing the parameters of a stack of $n_{\text {img }}$ particle holograms.
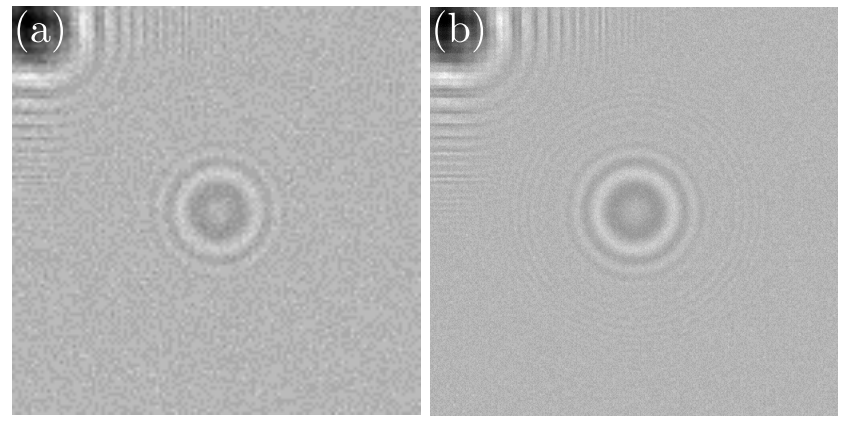

Fig. 3. Illustration of the super-resolution by IP approach with joint optimization. (a) Simulated low resolution hologram ; a Gaussian white noise has been added so that the $\mathrm{SNR}$ of hologram is 5 . The image area represents $128 \times 128$ pixels. (b) Hologram super-resolved by a factor 4 using the proposed method (given for illustration purposes).

Validation of the concept is proposed through numerical simulation of particle holograms. Here, $n_{\text {img }}=20$ holograms with both a moving and a fixed object are considered. The moving object consists of a $100 \mu \mathrm{m}$ diameter particle with random sub-pixel shifts between each simulated frame, whereas the fixed object is an opaque square. Joint optimization of the simulated sequence allows both estimation of $\{z, r\}$ and of the object shifts from one frame to the next. Moreover, independent optimization over each holograms as well a standard pixel SR method are considered for comparison. This latter method, is tested using an algorithm based on a global estimation of the shift from one frame to the next in the Fourier space [17]. The estimation of the parameters is then performed by analysis of the super- 
resolved hologram by IP approach.

A stack of $n_{\text {img }}=201024 \times 1024$ pixel low-resolution holograms are simulated according to the experimental conditions shown in Fig. 2. A Gaussian white noise has been added to the simulated hologram resulting in a SNR of 5 . A close-up view over $128 \times 128$ pixels is proposed Fig. 3(a).

Table 1. Comparison of particle parameter estimation, with simulated hologram sequences, for standard pixel SR method, independent and joint estimation.

\begin{tabular}{ccccc}
\hline SR method & $z_{\text {mean }}(\mathrm{mm})$ & $\sigma_{z}(\mu \mathrm{m})$ & $r_{\text {mean }}(\mu \mathrm{m})$ & $\sigma_{r}(\mu \mathrm{m})$ \\
\hline VandeWalle & 235.02 & 12.02 & 50.54 & 0.135 \\
IP approach & 235.01 & 14.41 & 49.98 & 0.094 \\
Joint IP & 235.01 & 3.16 & 49.98 & 0.019 \\
\hline Exact values & 235 & n.a & 50 & n.a \\
\hline
\end{tabular}

For visualization purposes, a super-resolved image is computed. The registration is performed using the estimated shifts, the hologram are interpolated to the desired SR factor value and superimposed. The maximal SR factor $S R_{\mathrm{f}}$ achievable can be linked to the number of acquired image by $S R_{\mathrm{f}} \approx \sqrt{n_{\mathrm{img}}} \approx 4.5$ [5]. Therefore, the SR factor is fixed to $S R_{\mathrm{f}}=4$. Results obtained are proposed Fig. 3. A close-up over $512 \times 512$ pixels of the super-resolved holograms is shown in Fig. 3(b). Here, the advantages of the approach are clearly visible. On one hand, higher spatial frequencies can be visualized on the super-resolved hologram, thereby increasing the accuracy of the reconstruction. On the other hand, the super-resolved hologram has a higher signal-to-noise ratio (SNR). Low SNR holograms can thus also benefit from this jointly optimized reconstruction method. Quantitative confirmation of this result can be found in the Table 1. Here, comparison between independent estimation over 1224 images, joint estimation over 61 stacks of 20 images, and super-resolved images based on standard pixel SR is realized. The improvement in the standard deviation of both $z$ and $r$ value is close to $\sqrt{n_{\text {img }}}$ as predicted by Robinson [5] in the case where optimized parameters are decorrelated. As seen in Table 1 Vandewalle's methods do not succeed in improving the standard deviation values. As a matter of fact, this method is based on a global motion estimation over the whole image, whereas only the object is moving. Let us note that our approach can be applied on stacks of images which present non subpixel shifts contrary to classical pixel SR schemes [17].

We here propose to take benefits of the jointly optimized IP approach reconstruction scheme for highaccuracy reconstruction of experimental holograms. The investigated object is a calibrated opaque chromium disk with a diameter of $100 \mu \mathrm{m}$ deposited on a glass substrate

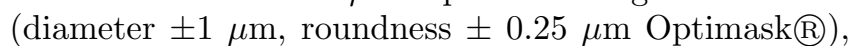
whose position is shifted using a 2-axis mechanical stage.
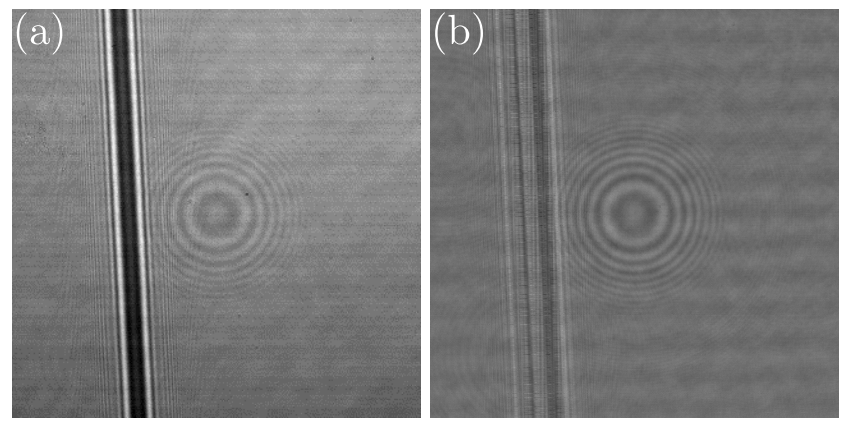

Fig. 4. Illustration of the super-resolution by IP approach with joint optimization. (a) Acquired low resolution hologram. The original image was cropped to $320 \times 320$ pixels. (b) Hologram super-resolved by a factor of 4 using the proposed method (given for illustration purposes).

The fixed object is a human hair. Low-resolution holograms are acquired over the complete area of the CMOS sensor. A close-up on the recorded low-resolution holograms over $320 \times 320$ pixels is presented Fig. 4(a). Part of the moving object interference pattern is occluded by the hair. Jointly optimized reconstruction by IP approach is performed over $n_{\text {img }}=20$ images with shifts of several tens of pixels between each acquisition. For illustration purposes, the super-resolved hologram with $S R_{\mathrm{f}}=4$ is presented in Fig. 4(b). It should be noted that the disturbance of the fixed object is weaker than that of the low-resolution hologram. This is due to the image registration step that results in averaging of the fixed
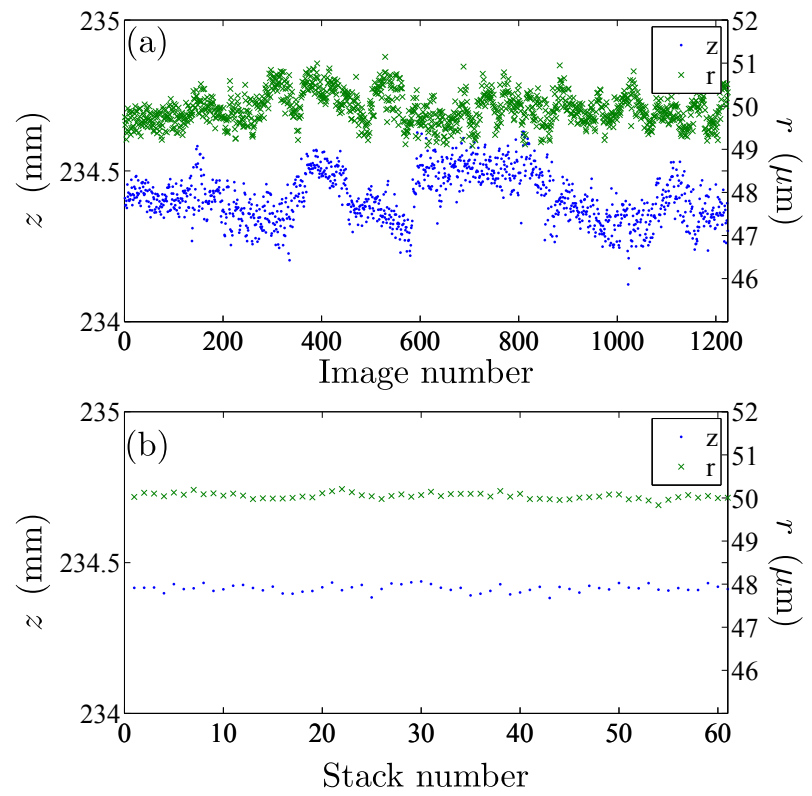

Fig. 5. (Color online) Evolution of the estimated $z$ (blue dots) and $r$ (green crosses) parameters according to the optimization scheme. (a) Individual IP approach over each acquired hologram. (b) Joint IP approach of the 61 image stacks. Each stack consists of 20 images. 
object signal over the sequence of holograms. As for the simulation results in Fig. 3, the super-resolved hologram contains higher spatial frequencies, that improve estimation of the object radius $r$, and object-to-sensor distance $z$.

The super-resolved holograms shown in Figs. 3 and 4 are given for illustration purposes and qualitatively demonstrate the benefits of using the proposed scheme. For quantitative assessment, comparison between a standard IP approach reconstruction method (i.e. optimization of the model parameters hologram by hologram), and the proposed joint optimization scheme is realized. A sequence of 1224 individual images (this amount corresponds to the camera buffer size) of the moving calibrated object are recorded with a frame-rate of $100 \mathrm{~Hz}$ and an exposure duration of $\tau_{\mathrm{e}}=6 \mu \mathrm{s}$. From these images, stacks of $n_{\text {img }}=20$ images are built by taking one frame out of 61 . According to the amount of acquired images, 61 image stacks are used to point out benefits of the approach. Values of $z$ and $r$ are optimized for each frame in the case of the classical IP approach and for each image stack for the joint IP approach. Obtained results are proposed Fig. 5. Here, estimated values of $z$ (blue dots) and $r$ (green crosses) for individual IP approach reconstruction (a), and joint IP approach reconstruction (b) are plotted versus either the frame number or the stack number.

One can notice that dispersion of $z$ and $r$ values is higher considering an individual processing scheme (Fig. 5(a)) rather than a joint scheme (Fig. 5(b)). This can be quantified by analyzing averaged values of $z$ and $r$ as well as their respective standard deviations $\sigma_{z}$ and $\sigma_{r}$. Obtained results are listed Table 2 and compared to manufacturer's specifications. The estimations of $z$ and $r$ are improved by more than a factor $\sqrt{n_{\mathrm{img}}}$. This is due to the existence of correlation between estimated parameters as discussed in Ref. [5]. This proves that the joint estimation provides in this case an improvement of the accuracy of the estimator. It should be noted, that $z$ values are slightly changed along the image sequence (probably due to the translation stage), thus leading to an overestimate of $\sigma_{z}$ (Fig. 5(a)). In order to be able to

Table 2. Comparison of particle parameter estimation, with experimental hologram sequences, for standard pixel SR method, independent and joint estimation.

\begin{tabular}{ccccc}
\hline SR method & $z_{\text {mean }}(\mathrm{mm})$ & $\sigma_{z}(\mu \mathrm{m})$ & $r_{\text {mean }}(\mu \mathrm{m})$ & $\sigma_{r}(\mu \mathrm{m})$ \\
\hline VandeWalle & 234.48 & 1240 & 52.85 & 1.513 \\
IP approach & 234.41 & 82.79 & 49.94 & 0.374 \\
Joint IP & 234.41 & 12.41 & 50.04 & 0.066 \\
\hline Manufacturer & n.a. & n.a & 50 & 1 \\
\hline
\end{tabular}

apply Vandewalle's method on our experimental holograms, the estimated particle shifts have been converted to subpixel shifts by means of an integer translation step.
Results obtained with a classical pixel SR scheme [17] have been calculated. They illustrate the inappropriateness of this method for our application. Indeed standard pixel SR schemes are based on a global estimation of the subpixel shifts from one frame to the next.

We have proposed a joint optimization method, applied to particle in-line hologram reconstruction, improving $z$ and $r$ estimation accuracy. It should be noted that this approach is valid as long as the imaging model is parametric. Benefits of the proposed scheme have been demonstrated with both numerical and bench-top experiments. Improvement of the accuracy on $z$ and $r$ have been proved to be higher than $\sqrt{n_{i m g}}$, thus reducing the gap between axial and lateral positioning accuracy. This approach can be generalized to high resolution 3D tracking considering joint estimation. For instance by estimating $\{x, y, z\}_{i=1: n_{\mathrm{img}}}$ and $r$ along an image sequence.

The authors are grateful for the financial support of Université de Lyon through the Program "Investissements d'Avenir" (ANR-1 1-IDEX-0007)

\section{References}

[1] J. Katz and J. Sheng, Annu. Rev. Fluid Mech. 42, 531 (2010).

[2] W. Bishara, T.-W. Su, A. F. Coskun, and A. Ozcan, Opt. Express 18, 11181 (2010).

[3] O. Mudanyali, E. McLeod, W. Luo, A. Greenbaum, A. F. Coskun, Y. Hennequin, C. P. Allier, and A. Ozcan, Nat. Photonics 7, 247 (2013).

[4] R. C. Hardie, K. J. Barnard, and E. E. Armstrong, IEEE T. Image Process. 6, 1621 (1997).

[5] D. Robinson and P. Milanfar, IEEE T. Image Process. 15, 1413 (2006).

[6] S. Sotthivirat and J. A. Fessler, J. Opt. Soc. Am. A 21, 737 (2004).

[7] D. J. Brady, K. Choi, D. L. Marks, R. Horisaki, and S. Lim, Opt. Express 17, 13040 (2009).

[8] Y. Rivenson, A. Stern, and B. Javidi, J. Disp. Technol. 6, 506 (2010).

[9] L. Denis, D. A. Lorenz, and D. Trede, Inverse Probl. 25, 115017 (2009).

[10] F. Soulez, L. Denis, C. Fournier, Éric Thiébaut, and C. Goepfert, J. Opt. Soc. Am. A 24, 1164 (2007).

[11] S. H. Lee, Y. Roichman, G. R. Yi, S. H. Kim, S. M. Yang, A. van Blaaderen, P. van Oostrum, and D. G. Grier, Opt. Express 15, 18275-18282 (2007).

[12] K. I. Mortensen, L. S. Churchman, J. A. Spudich, and H. Flyvbjerg, Nat. Methods 7, 377 (2010).

[13] C. Fournier, L. Denis, and T. Fournel, J. Opt. Soc. Am. A 27, 1856 (2010).

[14] S. M. Kay, Fundamentals of statistical signal processing: estimation theory, (Prentice-Hall, 1993).

[15] G. Tyler and B. Thompson, J. Mod. Opt. 23, 685 (1976).

[16] D. Chareyron, J.-L. Marié, C. Fournier, J. Gire, N. Grosjean, L. Denis, M. Lance, and L. Méès, New J. Phys. 14, 043039 (2012).

[17] P. Vandewalle, S. Süsstrunk, and M. Vetterli, EURASIP J. Adv. Sig. Pr., 071459 (2006). 


\section{References}

[1] J. Katz and J. Sheng, "Applications of holography in fluid mechanics and particle dynamics," Annu. Rev. Fluid Mech. 42, 531-555 (2010).

[2] W. Bishara, T.-W. Su, A. F. Coskun, and A. Ozcan, "Lensfree on-chip microscopy over a wide field-of-view using pixel super-resolution," Opt. Express 18, 1118111191 (2010).

[3] O. Mudanyali, E. McLeod, W. Luo, A. Greenbaum, A. F. Coskun, Y. Hennequin, C. P. Allier, and A. Ozcan, "Wide-field optical detection of nanoparticles using on-chip microscopy and self-assembled nanolenses," Nat. photonics 7, 247-254 (2013).

[4] R. C. Hardie, K. J. Barnard, and E. E. Armstrong, "Joint MAP registration and high-resolution image estimation using a sequence of undersampled images," IEEE T. Image Process. 6, 1621-1633 (1997).

[5] D. Robinson and P. Milanfar, "Statistical performance analysis of super-resolution," IEEE T. Image Process. 15, 1413-1428 (2006).

[6] S. Sotthivirat and J. A. Fessler, "Penalized-likelihood image reconstruction for digital holography," J. Opt. Soc. Am. A 21, 737-750 (2004).

[7] D. J. Brady, K. Choi, D. L. Marks, R. Horisaki, and S. Lim, "Compressive holography," Opt. Express 17, 13040-13049 (2009).

[8] Y. Rivenson, A. Stern, and B. Javidi, "Compressive fresnel holography," J. Disp. Technol. 6, 506-509 (2010).

[9] L. Denis, D. A. Lorenz, and D. Trede, "Greedy solution of ill-posed problems: error bounds and exact inversion," Inverse Probl. 25, 115017 (2009).
[10] F. Soulez, L. Denis, C. Fournier, Éric Thiébaut, and C. Goepfert, "Inverse-problem approach for particle digital holography: accurate location based on local optimization," J. Opt. Soc. Am. A 24, 1164-1171 (2007).

[11] S. H. Lee, Y. Roichman, G. R. Yi, S. H. Kim, S. M. Yang, A. van Blaaderen, P. van Oostrum, and D. G. Grier, "Characterizing and tracking single colloidal particles with video holographic microscopy," Opt. Express 15, 18275-18282 (2007).

[12] K. I. Mortensen, L. S. Churchman, J. A. Spudich, and H. Flyvbjerg, "Optimized localization analysis for single-molecule tracking and super-resolution microscopy," Nat. Methods 7, 377-381 (2010).

[13] C. Fournier, L. Denis, and T. Fournel, "On the single point resolution of on-axis digital holography," J. Opt. Soc. Am. A 27, 1856-1862 (2010).

[14] S. M. Kay, "Fundamentals of statistical signal processing: estimation theory," (Prentice-Hall, 1993).

[15] G. Tyler and B. Thompson, "Fraunhofer holography applied to particle size analysis a reassessment," J. Mod. Opt. 23, 685-700 (1976).

[16] D. Chareyron, J.-L. Marié, C. Fournier, J. Gire, N. Grosjean, L. Denis, M. Lance, and L. Méès, "Testing an in-line digital holography 'inverse method'for the lagrangian tracking of evaporating droplets in homogeneous nearly isotropic turbulence," New J. Phys. 14, 043039 (2012).

[17] P. Vandewalle, S. Süsstrunk, and M. Vetterli, "A frequency domain approach to registration of aliased images with application to super-resolution," EURASIP J. Adv. Sig. Pr., 071459 (2006). 